\title{
Evaluating lead halide perovskite nanocrystals as a spin laser gain medium
}

Beibei Tang ${ }^{\sharp, a, b, c}$, Guihai Lit ${ }^{\sharp a, b, c}$, Xuechen $R u^{d, e}$, Yan Gao ${ }^{f, g, h}$, Zidu Li ${ }^{a, b, c}$, Huaibin Shen ${ }^{f, g, h}$, Hong-bin Yao ${ }^{d, e}$, Fengjia Fan ${ }^{* a, b, c}$, Jiangfeng Du* ${ }^{*, a, b, c}$

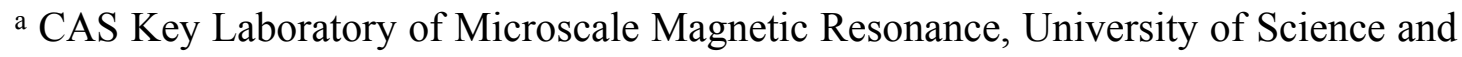
Technology of China, Hefei 230026, China

b School of Physical Sciences, University of Science and Technology of China, Hefei 230026, China

${ }^{\mathrm{c}}$ CAS Center for Excellence in Quantum Information and Quantum Physics, University of Science and Technology of China, Hefei 230026, China

${ }^{\mathrm{d}}$ Department of Applied Chemistry, University of Science and Technology of China, Hefei 230026, China

${ }^{\mathrm{e}}$ Hefei Science Center of Chinese Academy of Sciences, University of Science and Technology of China, Hefei, 230026, China

${ }^{\mathrm{f}}$ Key Laboratory for Special Functional Materials of Ministry of Education, Henan University, Kaifeng 475004, China 
g National \& Local Joint Engineering Research Center for High-efficiency Display and Lighting Technology, Henan University, Kaifeng 475004, China

${ }^{\mathrm{h}}$ Collaborative Innovation Center of Nano Functional Materials and Applications, Henan University, Kaifeng 475004, China

*Correspondence and requests for materials should be addressed to Fengjia Fan (ffj@ustc.edu.cn) and Jiangfeng Du (djf@ustc.edu.cn) 


\section{Methods}

Synthesis of $\mathrm{CsPbBr}_{3}$ NCs. The synthesis of $\mathrm{CsPbBr}_{3}$ NCs followed the previous reports $^{1-2} .95 \mathrm{mg} \mathrm{CH}{ }_{3} \mathrm{COOCs}, 380 \mathrm{mg} \mathrm{Pb}\left(\mathrm{CH}_{3} \mathrm{COO}\right)_{2} \bullet 3 \mathrm{H}_{2} \mathrm{O}, 1.5 \mathrm{ml}$ oleic acid (OA), $5 \mathrm{ml}$ oleylamine (OAm), and $25 \mathrm{ml}$ octadecene (ODE) were added into a three-necked flask. Then the mixture was degassed at $120^{\circ} \mathrm{C}$ for $15 \mathrm{~min}$ and filled with nitrogen for $10 \mathrm{~min}$. The process was repeated three times. After heating the mixture to $170{ }^{\circ} \mathrm{C}, 170$ ul benzoyl bromide was rapidly injected. Then the reaction mixture was cooled by an ice bath.

Isolation and purification of $\mathrm{CsPbBr}_{3}$ NCs. The synthesized NCs were separated by centrifuge and methyl acetate was added to the solution to help them to precipitate completely. After centrifugation, the NCs were dispersed in hexane.

Synthesis of $\mathrm{Cd}_{\mathrm{x}} \mathrm{Zn}_{1-\mathrm{x}}$ Se NCs. The $\mathrm{Cd}_{\mathrm{x}} \mathrm{Zn}_{1-\mathrm{x}}$ Se cores were prepared using a modified literature procedure ${ }^{3} .60 \mathrm{mmol} \mathrm{ZnO}, 60 \mathrm{~mL}$ OA, and $90 \mathrm{~mL}$ ODE were added into a $250 \mathrm{~mL}$ three-necked flask and degassed at $150{ }^{\circ} \mathrm{C}$ for 15 minutes. Then the mixture was heated to $310^{\circ} \mathrm{C}$ under nitrogen while stirring vigorously to obtain $\mathrm{Zn}(\mathrm{OA})_{2}$ precursor. For the growth of $\mathrm{Cd}_{\mathrm{x}} \mathrm{Zn}_{1-\mathrm{x}} \mathrm{Se}$ cores, $1.5 \mathrm{mmol} \mathrm{CdO}, 1.2 \mathrm{mmol} \mathrm{Zn}(\mathrm{OA})_{2}$ precursor, $20 \mathrm{~mL}$ ODE, and $7 \mathrm{~mL}$ OA were added into a $100 \mathrm{~mL}$ round flask. The mixture was heated to $150{ }^{\circ} \mathrm{C}$ and degassed at a pressure of 0.1 Torr for 15 minutes, filled with $\mathrm{N}_{2}$ gas, and heated to $300{ }^{\circ} \mathrm{C}$ to form a mixed solution of $\mathrm{Cd}(\mathrm{OA})_{2}$ and $\mathrm{Zn}(\mathrm{OA})_{2}$. At this temperature, $5 \mathrm{~mL}$ of degassed Se was swiftly injected into the reaction flask and reacted for $60 \mathrm{~min}$ to grow the core. For the overcoating of the $\mathrm{ZnS}$ shell, $\mathrm{Zn}(\mathrm{OA})_{2}$ and degassed $\mathrm{S}$ were dropped into the reaction solution using a syringe 
pump at a rate of $5 \mathrm{~mL} / \mathrm{h}$. The thickness of the shell could be controlled by the adding volume of the degassed $\mathrm{S}$. The solution was annealed at $300{ }^{\circ} \mathrm{C}$ for 20 minutes and cooled to room temperature after the reaction is complete.

Isolation and purification of $\mathbf{C d}_{\mathbf{x}} \mathbf{Z n}_{1-\mathrm{x}}$ Se NCs. The NCs were purified six times by the precipitation/dispersion method with a solvent combination of hexane/ ethanol (1/1 in volume ratio). The purified NCs were dispersed into hexane or chloroform for optical characterizations.

Transmission electron microscopy (TEM) measurement. TEM characterizations of $\mathrm{CsPbBr}_{3} \mathrm{NCs}$ and $\mathrm{Cd}_{\mathrm{x}} \mathrm{Zn}_{1-\mathrm{x}}$ Se NCs were performed on HT-7700 (100 kV, Hitachi) and JEM-2100 (200 kV, JEOL), respectively. NC dispersions were deposited on the copper meshes and placed on the sample stage after the solvent evaporated. The electrons emitted from the electron gun were accelerated and incident on the sample. After the electron passed through the sample, it was magnified and analyzed by the imaging system, then the microscopic morphology of the sample was obtained.

Absorbance measurement. The test wavelength of the UV-Vis spectrophotometer (P4PC, Mapada) was set as $350-750 \mathrm{~nm}$. The films were made by spin-coating the nanocrystals onto the glass slide at a spinning speed of $1000 \mathrm{rpm}$.

Photoluminescence (PL) measurement. A home-built confocal microscope was used to obtain the PL spectra of the film. It was excited by a high-frequency pulse laser (405 $\mathrm{nm}, 10 \mathrm{MHz})$. The spectra were collected and analyzed by an Ocean Optics spectrometer (FLMS12313). 
PL lifetime measurement. The photo-excitation setup of PL decay measurement was similar to the PL measurement. A high-frequency pulse (405 nm, $10 \mathrm{MHz}$ ) was used to excite the sample. The photons emitted from the sample were detected by an avalanche photodiode (APD) to generate a pulse signal, and then the arrival time of photons was obtained using a time-amplitude converter (TAC) triggered by the laser. Finally, a multi-channel analyzer (MCA) was used to obtain the photon number of different time channels. The PL lifetime was obtained by fitting the decay of the photon number to 1/e of the original value.

ASE measurement. A $480 \mathrm{~nm}$ femtosecond pulse (10 kHz, $190 \mathrm{fs})$ was focused on the sample. A fiber-coupled Spectrometer (FLMS12313, Ocean Optics) was used to collect spectra of samples under different photo-excitation powers. When the photo-excitation power was higher than the threshold value, the spectral width decreased and the intensity increased significantly.

Transient absorption (TA) measurement. TA spectroscopy measurements were performed on a Time-Tech Spectra (TA100) transient absorption spectrometer. The $1030 \mathrm{~nm}$ femtosecond pulse (10kHz, $190 \mathrm{fs})$ generated by a Yb:KGW laser (PHAROS, Light Conversion) is split proportionally into probe beam (10\%) and pump beam (90\%). After passing through a delay stage, the probe beam is focused on a BBO crystal and sapphire crystal to produce a white light continuum in the wavelength range of 400-550 $\mathrm{nm}$. Then the optical parametric amplifier (ORPHEUS-HE, Light Conversion) converts the pump beam into a laser pulse with a continuously tunable wavelength. The pump beam is incident on the sample at a small angle with the probe beam after passing through a chopper. The transmitted probe is collected and sent to the charge-coupled device and monochromator. The collected signal is the difference in probe intensity without and with the pump beam. According to the absorption spectrum, the probe 
intensity absorbed by the sample is obtained. Combining with the TA signal, the value of optical gain can be calculated from the original probe intensity and the probe intensity when the pumping beam is present.

Cross-section measurement. The cross-section measurement was based on the previous reports ${ }^{4} .200 \mu \mathrm{L} \mathrm{CsPbBr}_{3}$ solution is diluted 10 times and used to measure the absorption spectrum. The NCs in $100 \mu \mathrm{L}$ solution are dissolved by nitric acid and diluted with $3 \mathrm{~mL}$ deionized water. According to the concentration of $\mathrm{Cs}^{+}$measured by inductively coupled plasma spectroscopy (ICP), the number of NCs per cubic centimeter could be determined. Then the cross-section can be calculated using the formula: $\sigma=2.303 \mathrm{~A} / \mathrm{cl}$, where $\mathrm{A}$ is the absorbance at $480 \mathrm{~nm}, \mathrm{c}$ and $\mathrm{l}$ is the number of NCs per cubic centimeter and the optical path of the cuvette, respectively. 


\section{Supplementary Figures}
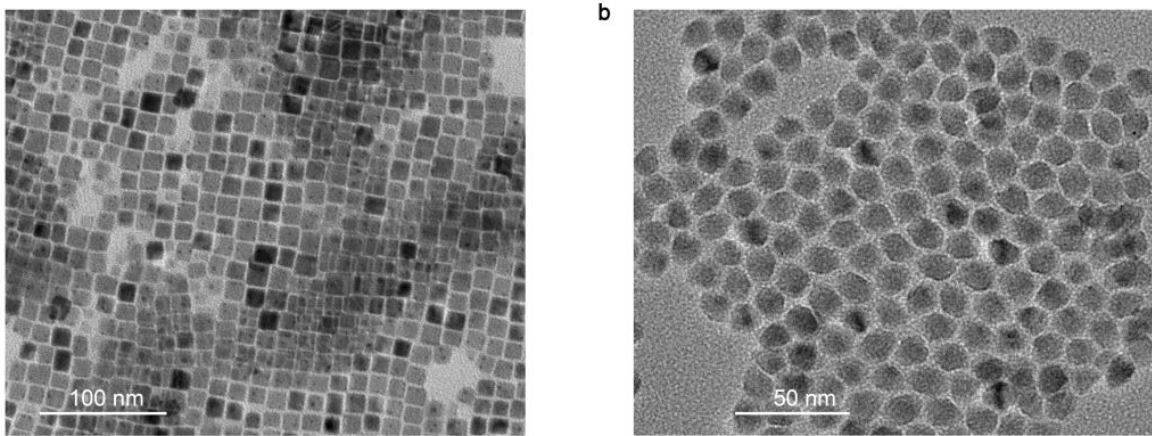

Figure S1. TEM images of (a) $\mathrm{CsPbBr}_{3} \mathrm{NCs}$ and (b) $\mathrm{Cd}_{\mathrm{x}} \mathrm{Zn}_{1-\mathrm{x}} \mathrm{Se} \mathrm{NCs}$, respectively.
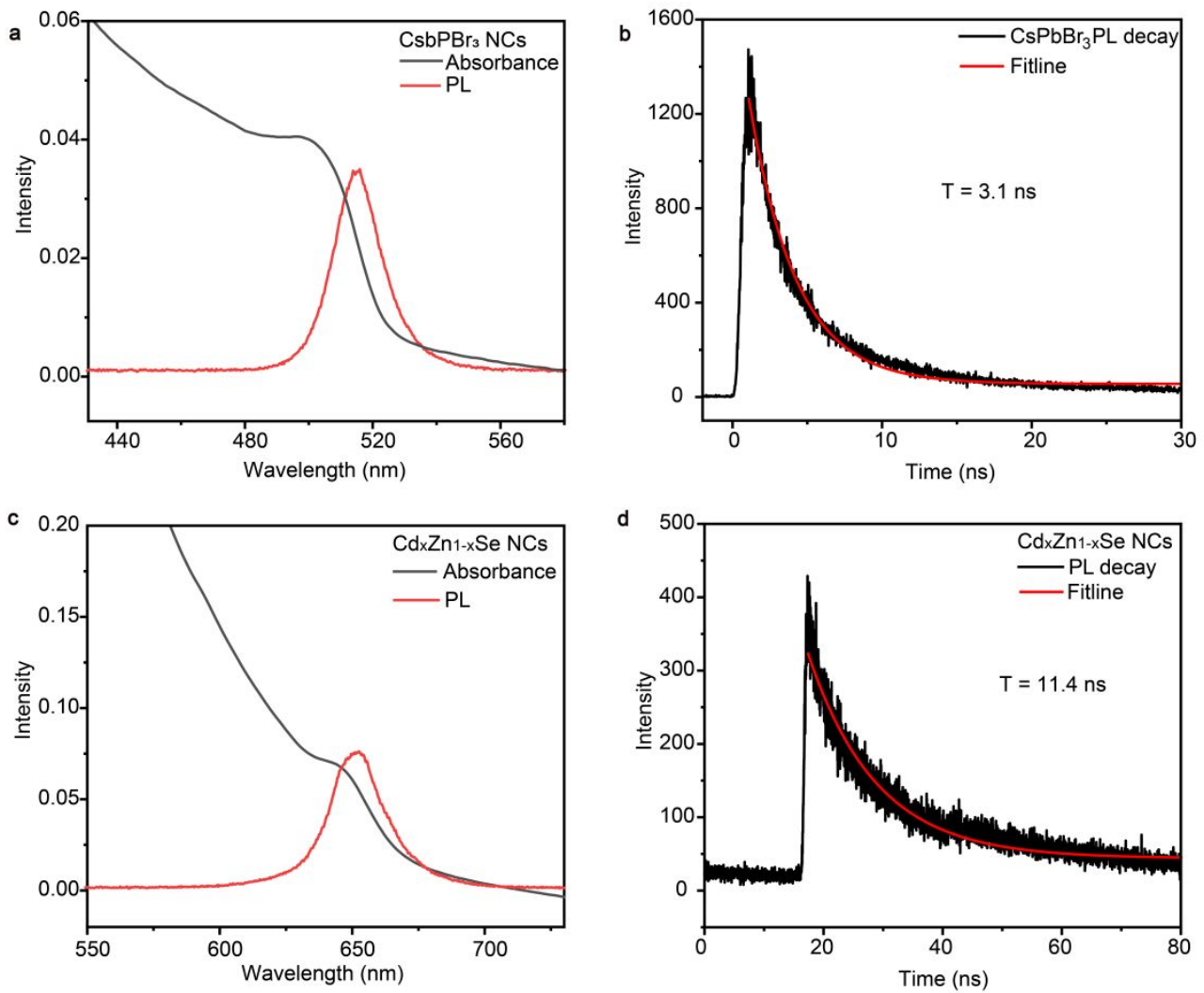

Figure S2. PL and absorbance spectra of (a) $\mathrm{CsPbBr}_{3} \mathrm{NCs}$ and (c) $\mathrm{Cd}_{\mathrm{x}} \mathrm{Zn}_{1-\mathrm{x}} \mathrm{Se} \mathrm{NCs}$, respectively. The lifetimes obtained from (b) $\mathrm{CsPbBr}_{3} \mathrm{NCs}$ and (d) $\mathrm{Cd}_{\mathrm{x}} \mathrm{Zn}_{1-\mathrm{x}} \mathrm{Se} \mathrm{NCs}$ by single exponential fitting are $3.1 \mathrm{~ns}$ and $11.4 \mathrm{~ns}$, respectively. 

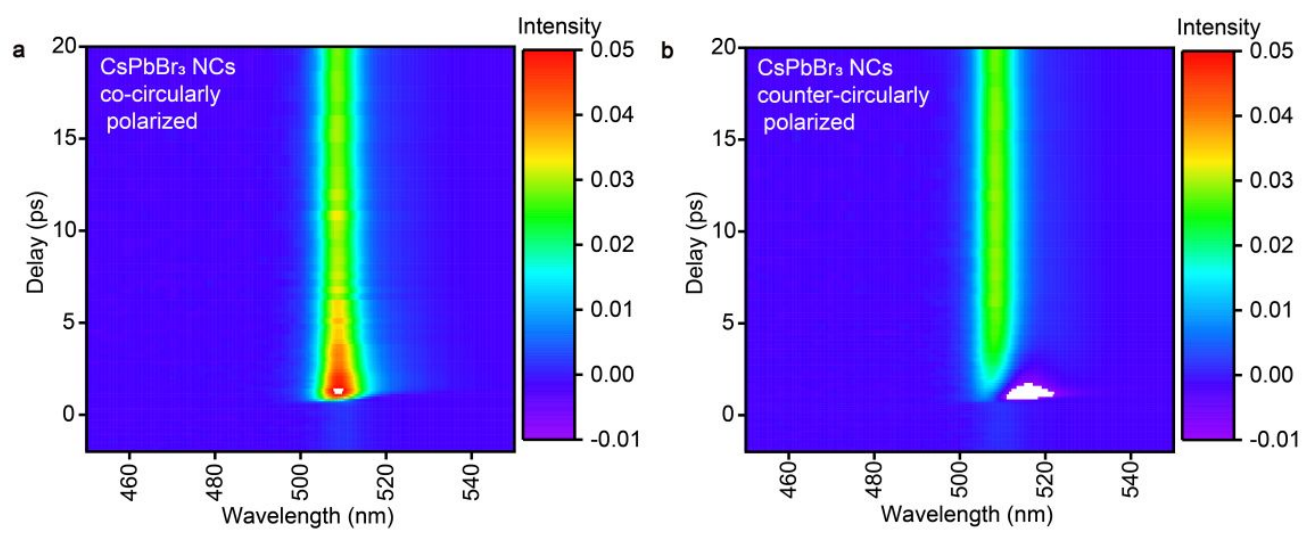

Figure S3. TA spectra of $\mathrm{CsPbBr} 3$ NCs under the (a) co-circularly and (b) the countercircularly polarized pump-probe. There is an obvious PIA signal in the first few picoseconds under the counter-circularly polarized pump-probe condition.
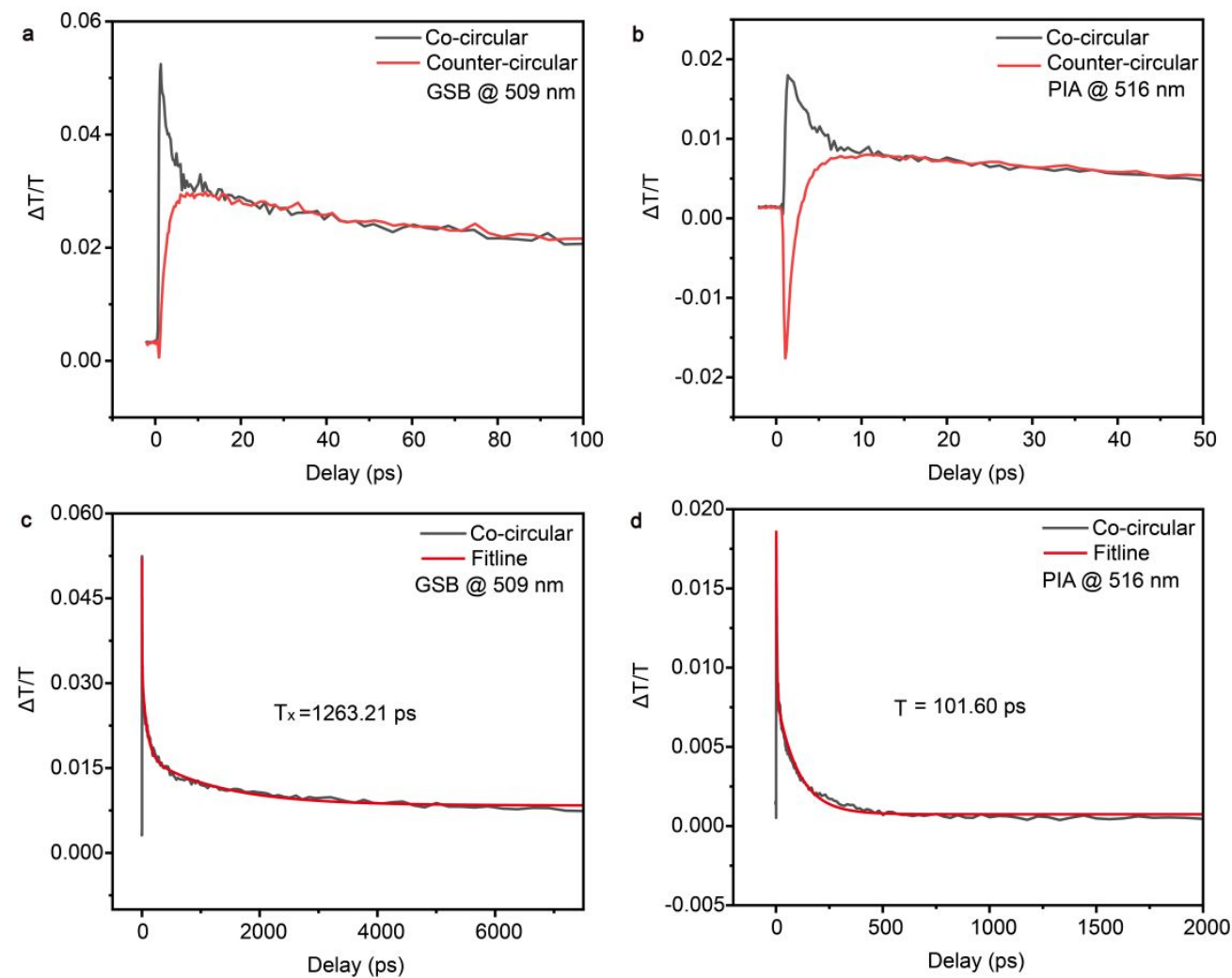

Figure S4. TA dynamics in different time ranges. (a) Bleach signal dynamics at 509 $\mathrm{nm}$ and (b) PIA signal dynamics at $516 \mathrm{~nm}$ were obtained under the co- and countercircularly polarized pump-probe within the first $100 \mathrm{ps}$. After $7 \mathrm{ps}$, two curves coincide. The longer decay dynamics of (c) GSB signal and (d) PIA signal with the co-circularly polarized pump-probe. The extracted exciton lifetime (red line) is about 1263.21 ps, and the lifetime of the PIA signal is $101.60 \mathrm{ps}$. 

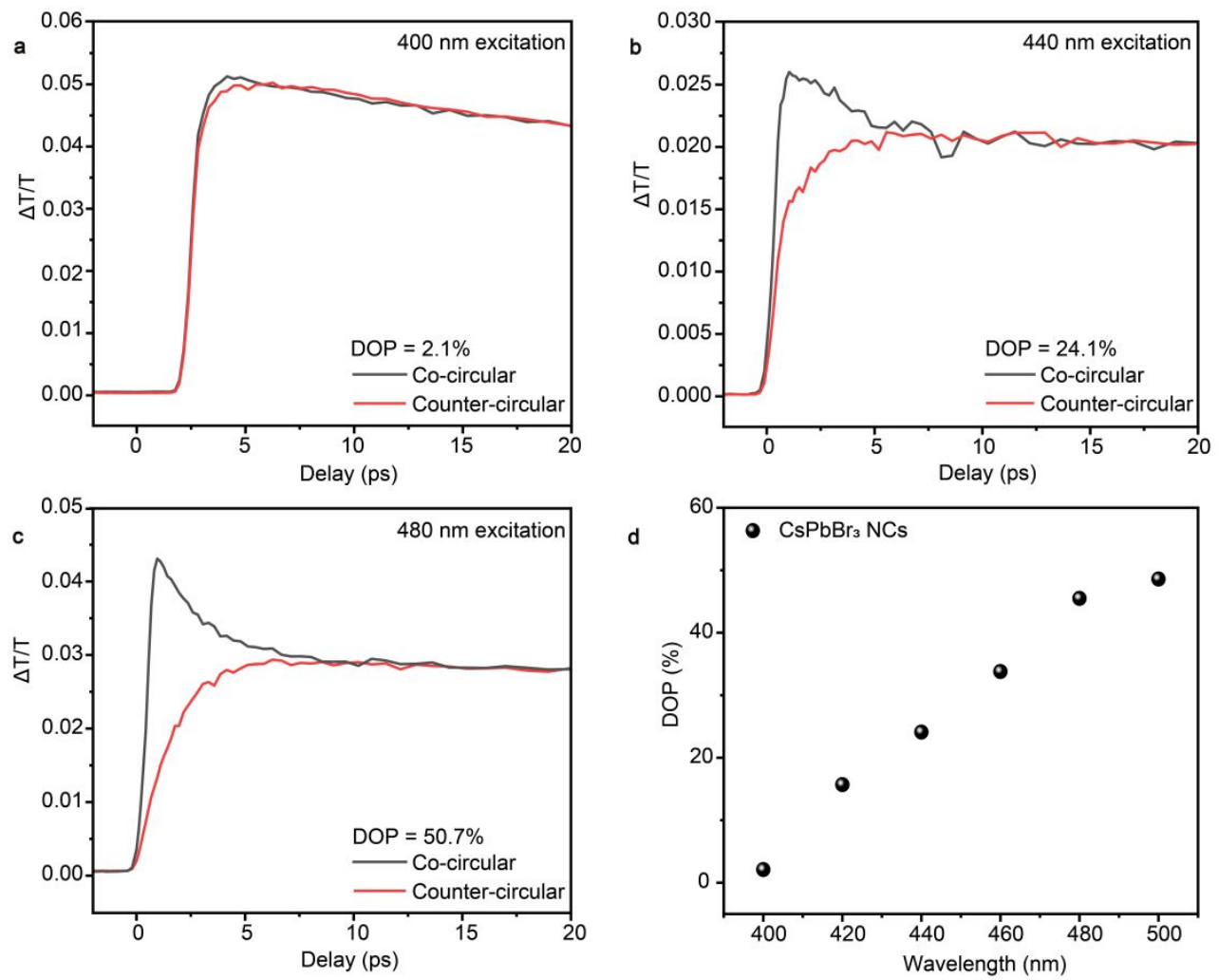

Figure S5. (a)-(c) TA spectra with photo-excitations at $400 \mathrm{~nm}, 440 \mathrm{~nm}$, and $480 \mathrm{~nm}$, respectively. (d) Wavelength dependent DOP of $\mathrm{CsPbBr}_{3} \mathrm{NCs}$. 

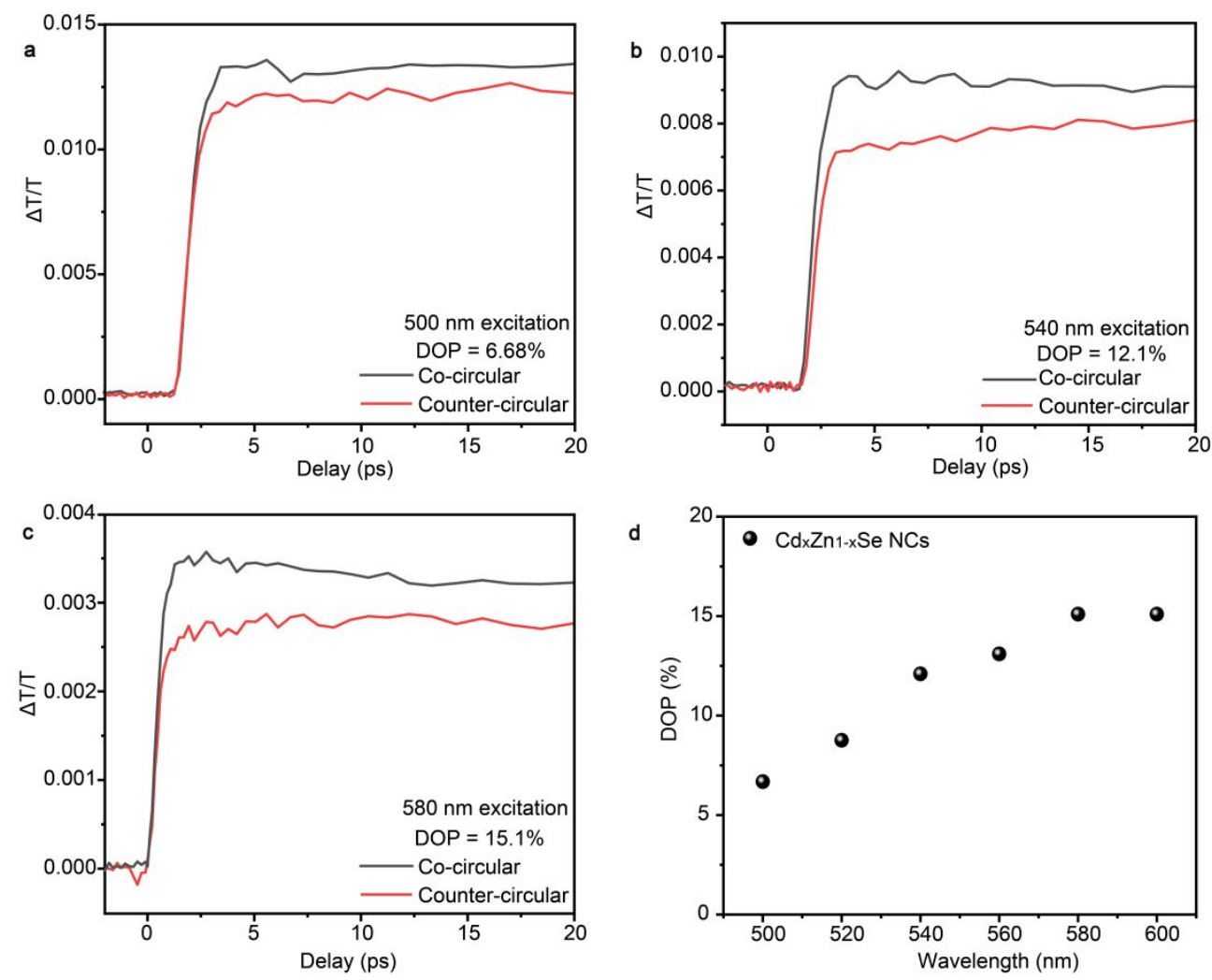

Figure S6. (a)-(c) TA spectra with photo-excitations at $500 \mathrm{~nm}, 540 \mathrm{~nm}$, and $580 \mathrm{~nm}$, respectively. (d) Wavelength dependent DOP of $\mathrm{Cd}_{\mathrm{x}} \mathrm{Zn}_{1-\mathrm{x}} \mathrm{Se} \mathrm{NCs}$.
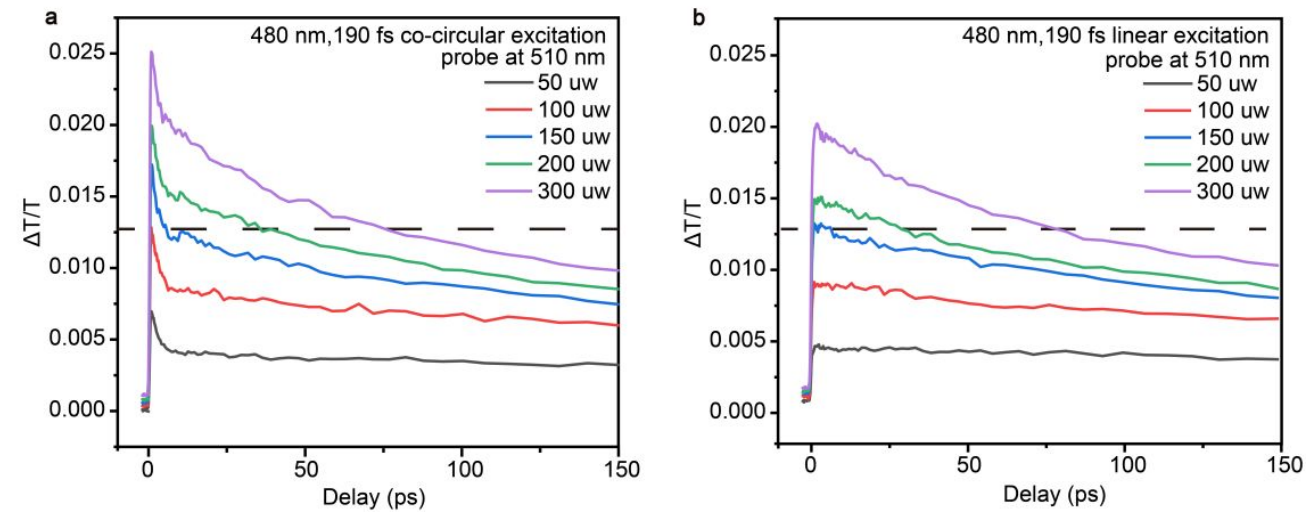

Figure S7. TA spectra under different photo-excitation intensity when $\mathrm{CsPbBr}_{3} \mathrm{NCs}$ are under circularly polarized (a) and linearly polarized (b) photo-excitation, respectively. 

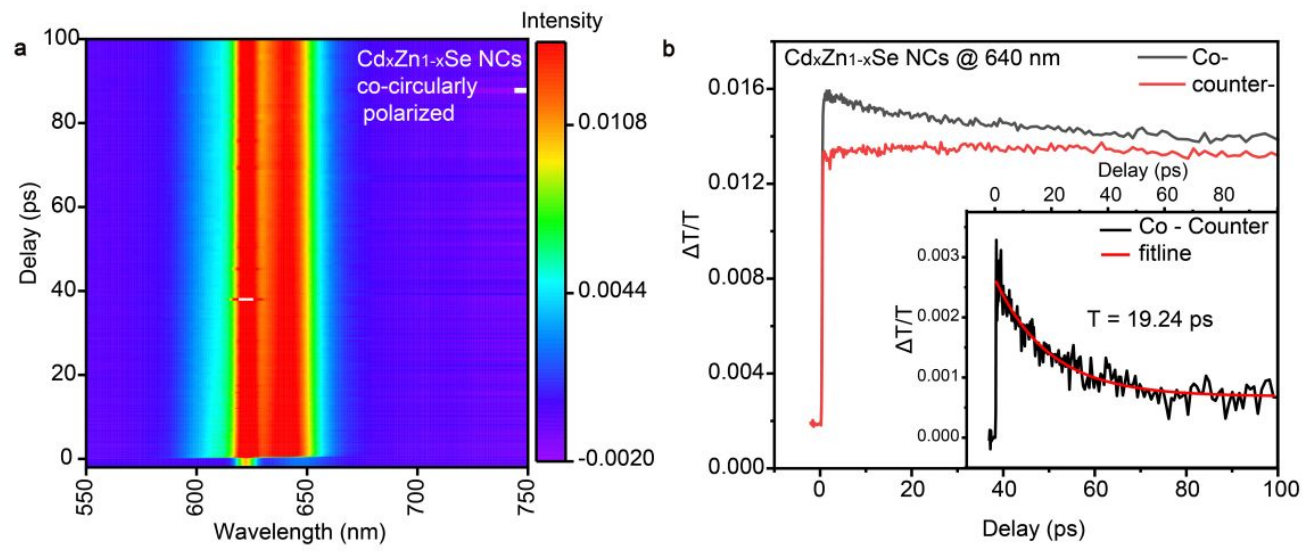

Figure S8. (a) Transient absorption spectra of $\mathrm{Cd}_{\mathrm{x}} \mathrm{Zn}_{1-\mathrm{x}} \mathrm{Se} \mathrm{NCs}$ under co-circularly polarized pump-probe configuration. (b) TA dynamics at $640 \mathrm{~nm}$ over a longer period (100 ps). The obtained DOP of $\mathrm{Cd}_{\mathrm{x}} \mathrm{Zn}_{1-\mathrm{x}} \mathrm{Se} \mathrm{NCs}$ is $12 \%$. The spin relaxation time extracted from the inset is $19.24 \mathrm{ps}$.

a
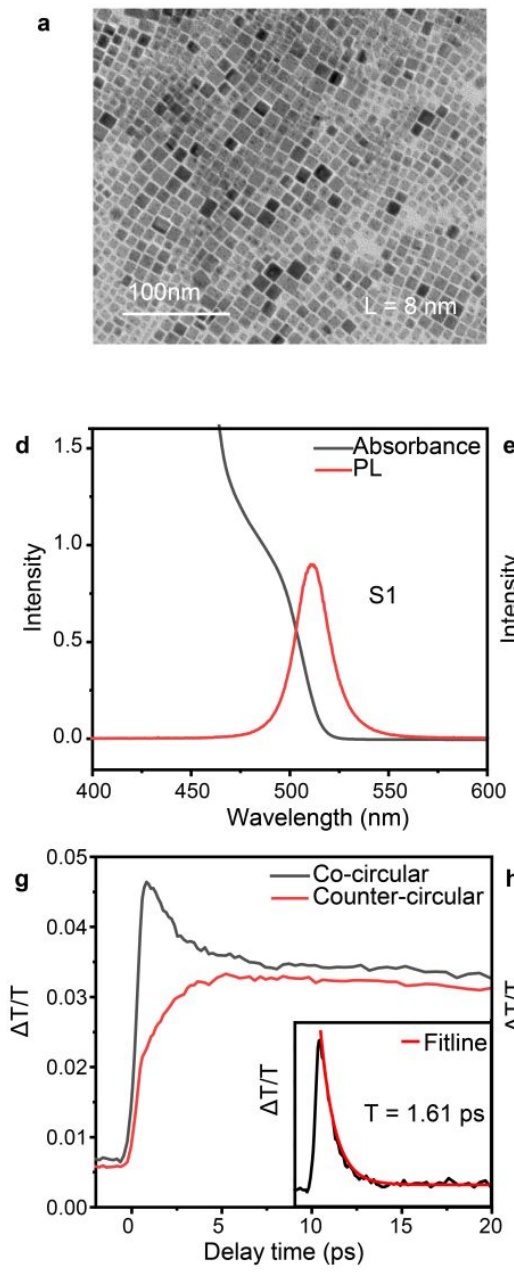

b
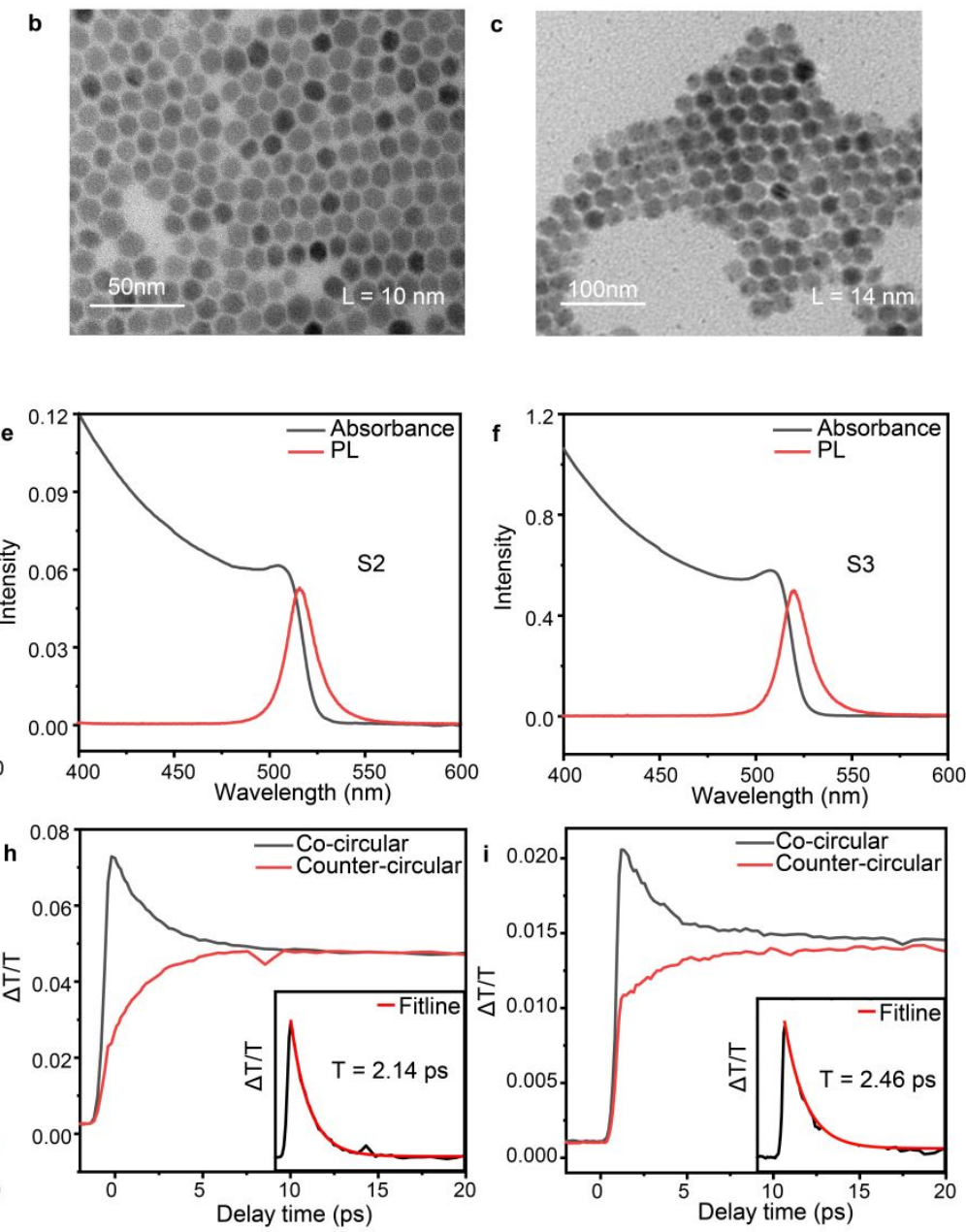
Figure S9. Size-dependent spin relaxation time measurements. (a)-(c) TEM images, (d)-(f) PL and absorbance spectra, and (g)-(i) TA spectra of $\mathrm{CsPbr}_{3} \mathrm{NCs}$ with different sizes, respectively. The spin relaxation time is extended with increasing size. The absorbance spectra were collected in solution samples and PL was measured from film samples spin-coated on glass.

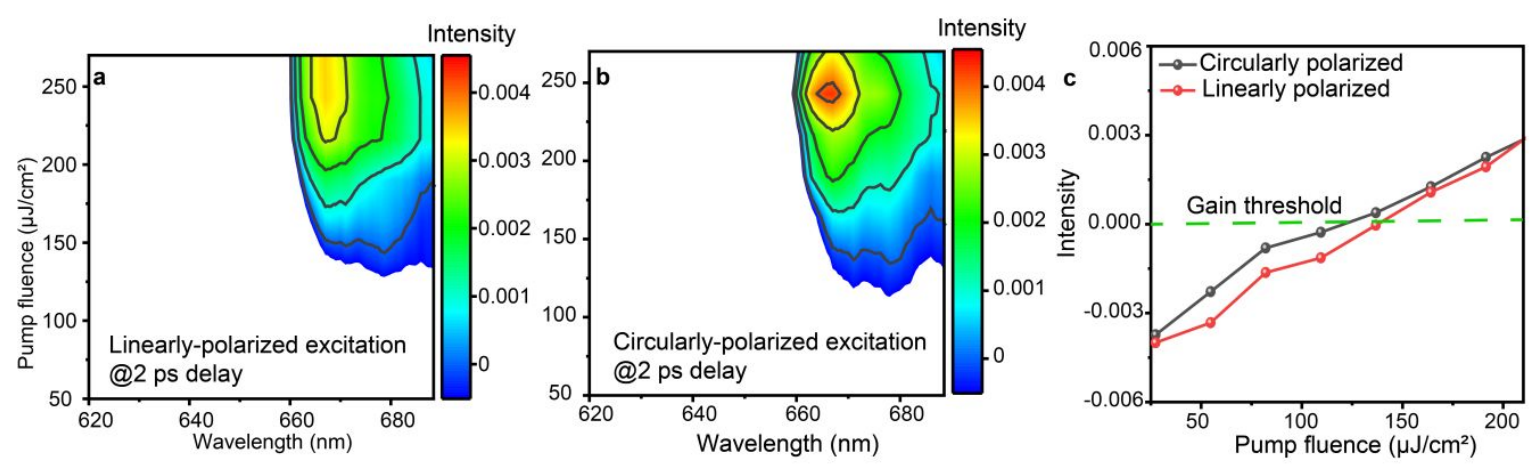

Figure S10. Polarization-dependent optical gain measurements of $\mathrm{Cd}_{\mathrm{x}} \mathrm{Zn}_{1-\mathrm{x}} \mathrm{Se} \mathrm{NCs}$. TA spectra were obtained at different pump fluences using the (a) circularly polarized and (b) unpolarized pump-probe, respectively. (c) the pump fluence dependence of GSB amplitude around $670 \mathrm{~nm}$.
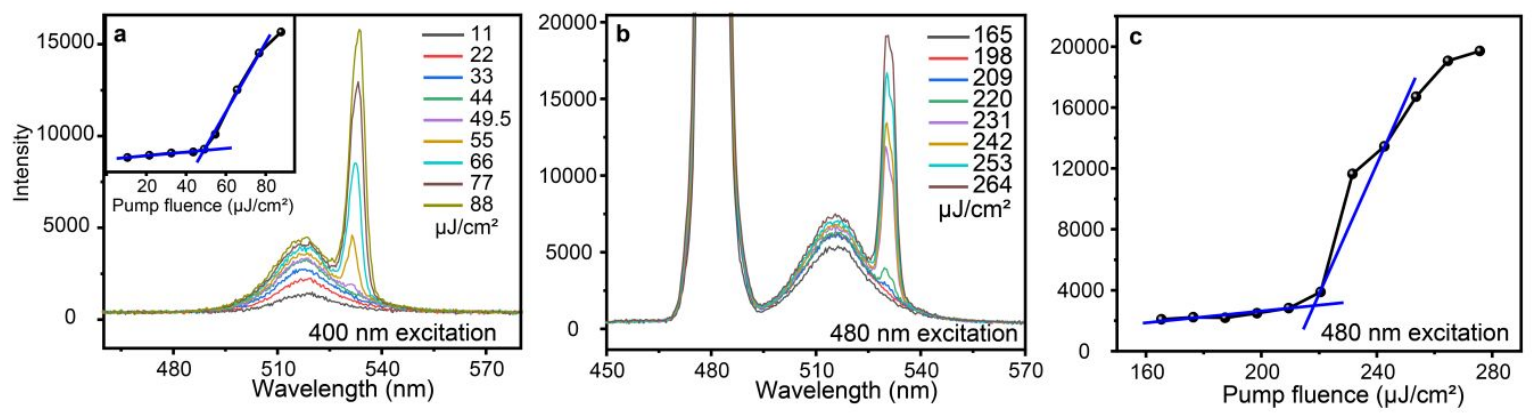

Figure S11. The threshold power measurements using (a) $400 \mathrm{~nm}$ and (b) $480 \mathrm{~nm}$ photoexcitation, respectively. (c) The dependence of peak intensity of ASE on pump fluence. 


\section{Supplementary Note}

Note S1. A detailed description of the theoretical simulation

We simulate the ASE process based on a previous report ${ }^{5-7}$. Based on the energy levels of perovskite nanocrystals, triplet exciton $\mathrm{J}=1(\mathrm{~m}=0, \pm 1)$ and singlet $\mathrm{J}=0$ exciton $^{8-9}$ are formed with electron-hole exchange interaction into consideration. Take Eqs.S1 as an example, we discuss the evolution process of NCs in different states after photo-excitation. The probability of NCs in the ground state decrease after absorbing the pump beam with different polarization at the initial stage. As the electron population changes, NCs in the triplet state return to the ground state. The probability of NCs in the ground state will increase due to the stimulated emission. In addition to the fundamental processes including absorption, radiative recombination, and stimulated emission, there are additional spin relaxation processes for NCs in the single exciton state. Therefore, the probability of NCs in other states and the evolution of the density of photons numbers over time can be obtained.

$$
\begin{gathered}
\frac{d P_{0}}{d t}=-P_{0} R_{\text {pump }}+\frac{J_{1,1}}{\tau_{X}}+\frac{J_{1,-1}}{\tau_{X}}+\frac{J_{1,0}}{\tau_{X}}+B_{X} \phi_{R}\left(J_{1,1}-P_{0}\right)+B_{X} \phi_{L}\left(J_{1,-1}-P_{0}\right)+ \\
B_{X} \phi_{0}\left(J_{1,0}-P_{0}\right) \\
\frac{d J_{1,1}}{d t}=P_{0} R_{\text {pump }} P+\frac{P_{X X}}{3 \tau_{X X}}-\frac{J_{1,1}}{\tau_{X}}+B_{X} \phi_{R}\left(P_{0}-J_{1,1}\right)+B_{X} \phi_{L}\left(P_{X X}-J_{1,1}\right)-\frac{J_{1,1}}{\tau_{1}} \\
\frac{J_{1,-1}}{3 \tau_{1}}+\frac{J_{1,0}}{3 \tau_{1}}+\frac{J_{0,0}}{3 \tau_{2}}+\frac{P_{X X}}{3 \tau_{a u}} \\
\frac{d J_{1,-1}}{d t}=-J_{1,-1} R_{p u m p} P+\frac{P_{X X}}{3 \tau_{X X}}-\frac{J_{1,-1}}{\tau_{X}}+B_{X} \phi_{R}\left(P_{X X}-J_{1,-1}\right)+B_{X} \phi_{L} \\
\left(P_{0}-\quad\right. \text { (S2) } \\
\left.J_{1,-1}\right)-\frac{J_{1,-1}}{\tau_{1}}+\frac{J_{1,1}}{3 \tau_{1}}+\frac{J_{1,0}}{3 \tau_{1}}+\frac{J_{0,0}}{3 \tau_{2}}+\frac{P_{X X}}{3 \tau_{a u}}
\end{gathered}
$$




$$
\begin{array}{r}
\frac{d J_{1,0}}{d t}=-J_{1,0} R_{\text {pump }}(1-P)+P_{0} R_{\text {pump }}(1-P)+\frac{P_{X X}}{3 \tau_{X X}}-\frac{J_{1,0}}{\tau_{X}}+B_{X} \phi_{0} \\
\left(P_{X X}-\quad J_{1,0}\right)+B_{X} \phi_{0}\left(P_{0}-J_{1,0}\right)-\frac{J_{1,0}}{\tau_{1}}+\frac{J_{1,1}}{3 \tau_{1}}+\frac{J_{1,0}}{3 \tau_{1}}+\frac{J_{0,0}}{3 \tau_{2}}+\frac{P_{X X}}{3 \tau_{a u}}
\end{array}
$$

$$
\begin{aligned}
\frac{d J_{0,0}}{d t}= & \frac{J_{1,1}}{3 \tau_{1}}+\frac{J_{1,0}}{3 \tau_{1}}+\frac{J_{1,-1}}{3 \tau_{1}}-\frac{J_{0,0}}{\tau_{2}} \\
\frac{d P_{X X}}{d t}= & J_{1,0} R_{p u m p}(1-P)+J_{1,-1} R_{p u m p} P-\frac{P_{X X}}{\tau_{X X}}+B_{X} \phi_{L}\left(J_{1,1}-P_{X X}\right)+ \\
& B_{X} \phi_{R}\left(J_{1,-1}-P_{X X}\right)+B_{X} \phi_{0}\left(J_{1,0}-P_{X X}\right)-\frac{P_{X X}}{\tau_{a u}} \\
\frac{d \phi_{R}}{d t}= & n_{0} B_{X} \phi_{R}\left(J_{1,1}-P_{0}\right)+n_{0} B_{X} \phi_{R}\left(P_{X X}-J_{1,-1}\right)-\frac{\phi_{R}}{\tau_{c}} \\
\frac{d \phi_{L}}{d t}= & n_{0} B_{X} \phi_{L}\left(J_{1,-1}-P_{0}\right)+n_{0} B_{X} \phi_{L}\left(P_{X X}-J_{1,1}\right)-\frac{\phi_{L}}{\tau_{c}} \\
\frac{d \phi_{0}}{d t}= & n_{0} B_{X} \phi_{0}\left(J_{1,0}-P_{0}\right)+n_{0} B_{X} \phi_{0}\left(P_{X X}-J_{1,0}\right)-\frac{\phi_{0}}{\tau_{c}} \\
B_{X}= & B \frac{h f}{\Delta f} \quad B=\frac{c^{3} \quad 1}{8 \pi h f^{3} \tau_{X}}
\end{aligned}
$$

Where $P_{0}, P_{X}\left(J_{1,0}, J_{1, \pm 1}, J_{0,0}\right)$ and $P_{X X}$ represents the probability of NCs in the ground state, the corresponding single exciton state, and the biexciton state, respectively. $R_{\text {pump }}$ is the pumping term. $B_{X}$ represents the rate of stimulated emission and stimulated absorption, and is obtained from the radiation lifetime by the Einstein relation. $f$ and $\Delta f$ represent the frequency and width of ASE peak, respectively. $\mathrm{h}$ and $c$ are Planck's constant and velocity of light in a vacuum. $\phi_{R}$ and $\phi_{L}$ are the density of photon numbers corresponding to left and right circular polarization, respectively. $\tau_{X}, \tau_{1}=\tau_{2}, \tau_{a u}, \tau_{X X}$, and $\tau_{c}$ represent the single exciton lifetime, spin relaxation time, Auger recombination lifetime, biexciton lifetime, and photon lifetime, respectively. $n_{0}$ is the number of NCs. 

Note S2. The interaction between band-edge fine structure and circularly polarized beams.

The band-edge fine structure of $\mathrm{CsPBr}_{3} \mathrm{NCs}$ consists of three orthogonally linear polarized states ${ }^{8}$, which can be expressed as:

$$
\begin{aligned}
& \left|\Psi_{X}\right\rangle=\frac{\mathbf{1}}{\sqrt{\mathbf{2}}}\left[|\Uparrow\rangle_{e}|\uparrow\rangle_{\boldsymbol{h}}-|\Downarrow\rangle_{\boldsymbol{e}} \mid \downarrow_{\boldsymbol{h}}\right] \\
& \left|\Psi_{Y}\right\rangle=\frac{1}{\sqrt{2}}\left[|\Uparrow\rangle_{e}|\uparrow\rangle_{h}+|\Downarrow\rangle_{e} \mid \downarrow_{h}\right] \\
& \left|\Psi_{Z}\right\rangle=\frac{1}{\sqrt{2}}\left[|\Downarrow\rangle_{e}|\uparrow\rangle_{h}+|\Uparrow\rangle_{e} \mid \downarrow_{h}\right]
\end{aligned}
$$

In which,

$$
\begin{aligned}
& |\Uparrow\rangle_{e}=-\frac{1}{\sqrt{3}}[(|X\rangle+i|Y\rangle)|\downarrow\rangle+|Z\rangle|\uparrow\rangle] \\
& |\Downarrow\rangle_{e}=\frac{1}{\sqrt{3}}[-(|X\rangle-i|Y\rangle)|\downarrow\rangle+|Z\rangle|\uparrow\rangle]
\end{aligned}
$$

and

$$
\begin{aligned}
& |\uparrow\rangle_{h}=|S\rangle|\uparrow\rangle \\
& |\downarrow\rangle_{h}=|S\rangle|\downarrow\rangle
\end{aligned}
$$

The optical excitation probability is proportional to the square of the matrix element. And the matrix elements can be expressed as

$$
\begin{aligned}
\left\langle 0|\hat{e} \cdot \hat{p}| \Psi_{X}^{e x}\right\rangle & \\
& =\int d^{3} r \frac{1}{\sqrt{2}} \\
& \left(\left\langleS \left|\langle\downarrow| \hat{e} \cdot \hat{p}\left(-\frac{1}{\sqrt{3}}(|X\rangle+i|Y\rangle)|\downarrow\rangle\right)+\left\langle S\left|\langle\uparrow| \hat{e} \cdot \hat{p}\left(-\frac{1}{\sqrt{3}}(|X\rangle-i|Y\rangle)|\uparrow\rangle\right)\right)\right.\right.\right.\right. \\
& =-\frac{2}{\sqrt{6}} \int d^{3} r\langle S|\hat{e} \cdot \hat{p}| \mathrm{X}\rangle
\end{aligned}
$$

Considering the interaction between right-circularly polarized light $\left(\hat{e}=\hat{e}_{x}-i \hat{e}_{y}\right)$ and a $\mathrm{CsPbBr}_{3} \mathrm{NC}$,

$$
\begin{aligned}
& \left\langle 0|\hat{e} \cdot \hat{p}| \Psi_{X}^{e x}\right\rangle=-\frac{2}{\sqrt{6}} \int d^{3} r\left\langle S\left|p_{x}\right| \mathrm{X}\right\rangle \\
& \left\langle 0|\hat{e} \cdot \hat{p}| \Psi_{Y}^{e x}\right\rangle=-\frac{2}{\sqrt{6}} \int d^{3} r\left\langle S\left|p_{y}\right| \mathrm{Y}\right\rangle
\end{aligned}
$$

When $\left\langle S\left|p_{x}\right| \mathrm{X}\right\rangle$ and $\left\langle S\left|p_{y}\right| \mathrm{Y}\right\rangle \neq 0$, the pump light can interact with the orthogonally linear states. 


\section{References}

(1) Protesescu, L.; Yakunin, S.; Bodnarchuk, M. I.; Krieg, F.; Caputo, R.; Hendon, C. H.; Yang, R. X.; Walsh, A.; Kovalenko, M. V., Nanocrystals of Cesium Lead Halide Perovskites (CsPbX(3), $\mathrm{X}=\mathrm{Cl}, \mathrm{Br}$, and I): Novel Optoelectronic Materials Showing Bright Emission with Wide Color Gamut. Nano Lett. 2015, 15 (6), 3692-3696.

(2) Raino, G.; Becker, M. A.; Bodnarchuk, M. I.; Mahrt, R. F.; Kovalenko, M. V.; Stoferle, T., Superfluorescence from lead halide perovskite quantum dot superlattices. Nature 2018, 563 (7733), 671-675.

(3) Shen, H.; Wang, H.; Zhou, C.; Niu, J. Z.; Yuan, H.; Ma, L.; Li, L. S., Large scale synthesis of stable tricolor $\mathrm{Zn}(1-\mathrm{x}) \mathrm{Cd}(\mathrm{x}) \mathrm{Se}$ core/multishell nanocrystals via a facile phosphine-free colloidal method. Dalton Trans 2011, 40 (36), 9180-9188.

(4) Fan, F.; Voznyy, O.; Sabatini, R. P.; Bicanic, K. T.; Adachi, M. M.; McBride, J. R.; Reid, K. R.; Park, Y. S.; Li, X.; Jain, A.; Quintero-Bermudez, R.; Saravanapavanantham, M.; Liu, M.; Korkusinski, M.; Hawrylak, P.; Klimov, V. I.; Rosenthal, S. J.; Hoogland, S.; Sargent, E. H., Continuous-wave lasing in colloidal quantum dot solids enabled by facet-selective epitaxy. Nature 2017, 544 (7648), 75-79.

(5) Yu, J. H.; Shendre, S.; Koh, W. K.; Liu, B. Q.; Li, M. J.; Hou, S. Y.; Hettiarachchi, C.; Delikanli, S.; Hernandez-Martinez, P.; Birowosuto, M. D.; Wang, H.; Sum, T.; Demir, H. V.; Dang, C., Electrically control amplified spontaneous emission in colloidal quantum dots. Sci. Adv. 2019, 5 (10), eaav3140.

(6) Wu, K.; Park, Y.-S.; Lim, J.; Klimov, V. I., Towards zero-threshold optical gain using charged semiconductor quantum dots. Nature Nanotechnology 2017, 12 (12), 1140-1148.

(7) Lim, J.; Park, Y.-S.; Klimov, V. I., Optical gain in colloidal quantum dots achieved with directcurrent electrical pumping. Nat. Mater. 2018, 17 (1), 42-50.

(8) Becker, M. A.; Vaxenburg, R.; Nedelcu, G.; Sercel, P. C.; Shabaev, A.; Mehl, M. J.; Michopoulos, J. G.; Lambrakos, S. G.; Bernstein, N.; Lyons, J. L.; Stoferle, T.; Mahrt, R. F.; Kovalenko, M. V.; Norris, D. J.; Raino, G.; Efros, A. L., Bright triplet excitons in caesium lead halide perovskites. Nature 2018, 553 (7687), 189-193.

(9) Sercel, P. C.; Lyons, J. L.; Wickramaratne, D.; Vaxenburg, R.; Bernstein, N.; Efros, A. L., Exciton Fine Structure in Perovskite Nanocrystals. Nano Lett. 2019, 19 (6), 4068-4077. 